\title{
Demonstration of optical communication network for ultra high-definition image transmission
}

\author{
- Proof-of-concept experiment of image distribution \\ over the dynamic optical path network -
}

\author{
Junya KURUMIDA * and Shu NAMIKI
}

[Translation from Synthesiology, Vol.4, No.2, p.100-110 (2011)]

\begin{abstract}
In order to overcome the limitation of low-power-consumption and communication capacity of the equipment forming the present communication network, National Institute of Advanced Industrial Science and Technology (AIST) has carried out a demonstration of a new prototype optical path network to support the super HD large-capacity image contents era, in collaboration with five IT- related companies and with the support of National Institute of Information and Communications Technology (NICT) and Nippon Hoso Kyokai Science and Technology Research Laboratories (NHK/STRL). The experiment was a trial to demonstrate the performance of the dynamic optical path network developed mainly by AIST under practical conditions, using a network testbed of field optical fiber links connecting Akihabara, Otemachi and Koganei offices in Tokyo. The demonstration included the cross-border-connection with the optical packet and circuit integrated network realized by NICT and transmission of super HD video signals developed by NHK. In this paper, we describe the objectives, targets, technology syntheses based on the elemental technologies for the demonstration, and outcomes.
\end{abstract}

Keywords : Optical path network, optical packet and circuit integrated network, JGN2plus, distribution of high-quality images, super high vision (SHV)

\section{Introduction}

With the background of increased demand in communication mainly for the Internet, energy savings in optical communication is an immediate issue. The most important topic of communication system R\&D fields today is to devise a network configuration that allows the viewers to obtain high-capacity information such as super high-definition (HD) images at home at their convenience, while maintaining low power consumption in the communication devices. Whenever a demand is made, the optical fiber path quickly switches to link the home and the information source to allow delivery of all sorts of information services. Such optically transparent network, where the home and the information source are connected by optical fiber only, is ideal because the communication bandwidth can be maximized to its theoretical limit without increasing the energy. This can then be used in various application technologies and services such as education, welfare, or medical care that employ super HD image transmission. Nowadays, the services based on the communication technology are handling large volumes of data, thus, high-speed data communication has become necessary. The optical fiber communication technology has a capability of handling such high-capacity data transmission, and it has become the foundation of the Internet and the advanced information society. On the other hand, there is a major problem in the sustainable development of such advanced communication services. It will be explained in the following chapter.

In August 2010, we conducted a demonstration experiment of the network that allows transmission of high-capacity information as typified by super HD videos on demand, by combining the elemental technologies for optical communication that were newly developed by AIST and five information technology companies. This enabled a proposal of the new network technology that overcomes the limitations of power consumption and communication capacity of the devices that configure the current network. In this paper, we describe the process of the technological considerations for synthesizing the individual elemental technologies and obtaining the functioning results.

\section{Social demand for optical network}

It is well known that the communication traffic on the Internet is increasing at the rate of $30 \sim 40 \%$ every year in Japan. Assuming that this trend continues for about 20 years, the traffic will be about 1,000 times higher. This calls for the improvement of power efficiency. The number of Japanese Internet users surpassed 94 million people at the end of 2009 , and the penetration rate is $78 \%^{[1]}$. The technological background is that the performances of the communication equipment and PDAs have improved, well-developed

Network Photonics Research Center, AIST Tsukuba Central 2, 1-1-1 Umezono, Tsukuba 305-8568, Japan * E-mail : j.kurumida@ aist.go.jp

Original manuscript received February 7, 2011, Revisions received March 31, 2011, Accepted April 6, 2011 
application software has become available, and a click on the computer (including mobile terminals) connected to the Internet can trigger transmission and reception of high-capacity data. Video information services are provided through the Internet, and the major issue now is how to realize a network while considering the power efficiency to deal with the increased communication traffic. Particularly, the configuration of the optical network consisting of the optical fiber communication technology that is capable of supporting high-capacity data communication is emphasized. The followings are the reasons for the demand based on the social background. Until now, the measures for increased Internet traffic included the parallel installation of communication devices or shifting to equipment with higher communication speed. However, the increased installation of the conventional communication equipment that employed high-speed electric signal processing linearly increased the power consumption as the communication speed increased (power issue). Also, as the connection technology of the optical subscriber lines advanced and optical fibers reached the homes, there has been increasing difficulty in efficiently concentrating the diversified broadband services to the communication node (base station) (communication capacity and network configuration issues). To solve these issues, the important point is how the optical communication lines are developed and configured into a true optical network.

The Network Photonics Research Center, AIST proposes a new network called the "optical path network" that directly links the users with the optical path, and engages in R\&D to solve the above issues. Particularly, the network where the optical network is switched flexibly to actively maintain the optical path in response to the high-capacity information request is called the "dynamic optical path network". Specifically, we engage in the technological developments for transmission/reception of the video images, silicon optical path switch, and dispersion compensation, to create an optical network that realizes the broadband service based on the appropriate management and control of each device and device group. Figure 1 shows the conceptual diagram. To utilize the optical switch in the network, a mechanism for controlling the optical switch according to the network application is necessary. That is to say, a mechanism is needed where the mutual connections are made between the HD video server and display, teleconference systems are handled, and these are appropriately managed according to the optical path and storage information. Moreover, vertical integration (collaboration) is essential to introduce the silicon optical path switch and wavelength resource management technologies, and to consolidate and realize the technologies that are developed individually, from device level to application level. These efforts will enable the high-speed data communication without going through multiple electric signal processing, and a network that handles large-capacity information at low power can be configured. In this paper, we mainly describe how the elemental technologies were built up into a system, and how the demonstration experiment was conducted.

When the issues manifested in the network are solved by stepby-step approach, it will provide great advantage socially and economically. This may become a communication infrastructure for various services, including the information provision for automobiles and robots (including remote operation), as well as teleconferencing and remote sensing. Such new communication infrastructure will generate new social values in terms of applications software. For example, teleconferencing in which three-dimensional images are shared will decrease the movement of people or business trips, and highly realistic HD video service will decrease the necessity for people to actually go to a certain place. High-speed high-capacity communication infrastructure may provide the basic technology that supports tele-existence, tele-presence, and tele-immersion.

\section{Scenario of the demonstration experiment}

In general, communication network is considered to be a social infrastructure equivalent to electricity, gas, and water. Usually, a new optical fiber network will not be installed from scratch in a city, except in areas where there is no existing network. Therefore, the new network must be implemented step by step by combining the existing and the new technologies. It is necessary to consider the incorporation of the super HD videos into the network for the future. Therefore, we shall discuss the issue from three perspectives. A) To incorporate the distribution of the super HD videos into the demo experiment, we think of a network configured by the coexistence of the super HD video distribution technology (bitrate $<43 \mathrm{~Gb} / \mathrm{s}$ ) and the elemental communication technologies for $1 \mathrm{~Gb} / \mathrm{s}$ or $10 \mathrm{~Gb} / \mathrm{s}$ that are already in commercial networks. There are two reasons for doing this. First is to demonstrate that the different communication rates can be handled simultaneously on the network, with focus on the super HD video distribution technology. Second is to demonstrate that it is possible to manage them on the network, to determine the services according to the request, and to actually switch the network for smooth distribution. Next, B) the configuration using the actual field optical fiber is considered. There are also two

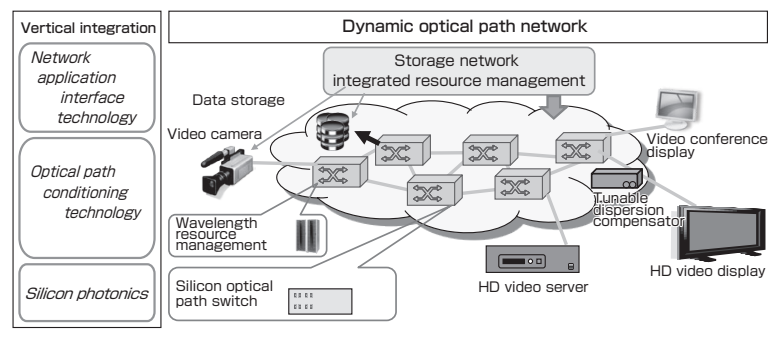

Fig. 1 Conceptual diagram of the dynamic optical path network 
reasons for this. One is to check that the communication is possible by compensating all the nonlinear responses from the field optical fiber, and the other is to check whether the different networks configured by different elements can be mutually connected. Also, some modifications are made to the entire system to $\mathrm{C}$ ) achieve low power consumption.

Above is the framework for the scenario of the demo experiment. The dynamic optical path network will be built based on this framework. One factor will be whether it is possible to achieve the high-speed high-capacity communication infrastructure geared for the near future by merely integrating and combining the aforementioned items. The aforementioned perspective mainly looks at the hardware, and that alone will not enable correct synthesis of the network. It is necessary to design the software and firmware to match the hardware. Figure 2 shows the configuration elements of the demo experiment. In the configuration elements, there are the following limiting conditions: to use the field optical fiber, to use the communication elemental technologies that we developed, and to add the resource management and control. Moreover there are two aspects of software and hardware for these conditions, and a consideration for a comprehensive configuration is necessary to conduct the demo experiment effectively.

The elemental technologies will be explained in the next chapter in more detail, but extra scenarios were considered in actually executing the demo. Although it might not be used in the demonstration, the network extendibility was added in the elemental technology as a backup in preparation of the worst-case situation. Such preparation is done not only in demonstration experiment, but is done in actual communication systems. Real systems are designed to be reconfigurable according to the status of the problem or have implemented functions that allow alternative functions. Considering the above, we created a scenario to demonstrate the mixed bitrates, mutual connection of heterogeneous networks, and provision of the HD video service according to requests.

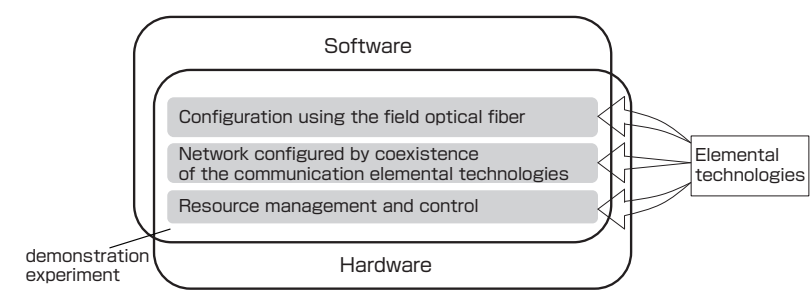

Fig. 2 Configuration of the demonstration experiment
Table 1. Configuration element of the demonstration experiment

\begin{tabular}{|c|c|c|c|c|}
\hline \begin{tabular}{|l|} 
Configuration \\
elements
\end{tabular} & Name of technologies & \begin{tabular}{|l} 
Collaboratiting \\
organizations
\end{tabular} & References & Notes \\
\hline \multirow{2}{*}{$\begin{array}{l}\text { Resource } \\
\text { management }\end{array}$} & (a) Optical path network & NTT / AIST & [2][3] & \\
\hline & $\begin{array}{l}\text { (b) Optical Packet and Circuit Integrated } \\
\text { Network }\end{array}$ & NICT & [4] & \\
\hline \multirow{2}{*}{\begin{tabular}{|l} 
Communication \\
line
\end{tabular}} & (c) R\&D testbed network & JGN2plus(NICT) & [5] & \\
\hline & $\begin{array}{l}\text { (d) Commercial optical fiber line (Akihabara - } \\
\text { Otemachi) }\end{array}$ & - & & \\
\hline \multirow{8}{*}{\begin{tabular}{|l} 
Node \\
equipment \\
and device
\end{tabular}} & (e) PLC optical switch & - & & $\begin{array}{l}\text { Commercially } \\
\text { available }\end{array}$ \\
\hline & (f) Silicon photonics switch & AIST & [6] & \\
\hline & \begin{tabular}{|l} 
(g) Current-injection-type silicon-based \\
high-speed optical switch
\end{tabular} & $\begin{array}{l}\begin{array}{l}\text { Fujitsu Laboratories } \\
\text { Ltd. }\end{array} \\
\end{array}$ & [7] & \\
\hline & (h) Power-saving next-generation ROADM & NEC & [8] & \\
\hline & (i) High-speed wavelength tunable laser & Trimatiz Ltd. & & \\
\hline & (j) Optical fiber amplifier (EDFA) & - & & $\begin{array}{l}\text { Commercially } \\
\text { available }\end{array}$ \\
\hline & (k) Parametric arbitrary wavelength converter & \begin{tabular}{|l} 
Furukawa Electric \\
Co., Ltd./ / AlST \\
\end{tabular} & [10] & \\
\hline & \begin{tabular}{|l} 
(I) High-speed autonomous-control tunable \\
dispersion
\end{tabular} & \begin{tabular}{|l} 
Furukawawa Electric \\
Co.. Ltd./ AlST \\
\end{tabular} & {$[11]=[13]$} & \\
\hline \multirow[t]{4}{*}{ Terminal } & (m)Super Hi-Vision transceiver & NHK & {$[14]$} & \\
\hline & (n) Contents achieve server & - & & General-use \\
\hline & Video delivery server & - & & General-use \\
\hline & Display (monitor) & - & & $\begin{array}{l}\text { Commercially } \\
\text { available }\end{array}$ \\
\hline \multirow[t]{2}{*}{ Contents } & (o) Super Hi-Vision image & NHK & & \\
\hline & (p) Hi-Vision video & AIST & & \\
\hline
\end{tabular}

\section{Elemental technologies}

The elemental technologies of the optical network demo experiment can be broken down into five parts. They are: network resource management, communication line as a medium through which the information is transmitted, nodes or equipment placed at their terminals, terminal device for transmitting/receiving, and the actual information (contents) that flow through the lines and devices. Table 1 shows the summary of the elemental technologies. In this chapter, the elemental technologies of configuration will be described according to Table 1, and the reasons for selecting them will be clarified.

\subsection{Resource management}

\subsubsection{Optical path network (NTT and AIST)}

The Network Photonics Research Center, AIST has engaged in the R\&D of optical path network, and developed the technology to optimize the optical fiber dispersion and optical intensity of the communication path, to eliminate the effect of the various optical switches that are used to switch the communication path ${ }^{[2]}$. In this demonstration, the PLC type optical switch ${ }^{\text {Term } 1}$ (Table 1(e)) was used for the node, which was connected by the optical fiber patch cord several meters long to form the optical path network.

The network and storage resource manager in the optical path network was realized by the collaboration between the Nippon Telegraph and Telephone (NTT) Corporation and the Information Technology Research Institute, AIST. The network resource manager was installed to manage the optical path and the resource manager that managed the reservation status of the performance-assured storage, and optimal server selection and optical path were set for the video on demand from the viewer ${ }^{[3]}$.When the reservation from the viewer was received and the reservation time arrived, the optical path opened to start the content 
distribution for the viewer. It was also designed to handle the multiple bitrate signals containing $1 \mathrm{~Gb} / \mathrm{s}, 10 \mathrm{~Gb} / \mathrm{s}$, and $43 \mathrm{~Gb} / \mathrm{s}$. The software that indicated the reservation status of the optical path was developed, and the user interface was provided at the same time.

\subsubsection{Mutual connection to the Optical Packet and Circuit Integrated Network (NICT)}

To demonstrate that the mutual servicing of heterogeneous network was possible, we conducted the demonstration jointly with the Optical Packet and Circuit Integrated Network of the National Institute of Information and Communication Technology (NICT). With the cooperation of NICT, the hand-over of the control signal was determined and was accomplished by exchanging the contents and service information at the connection node. High-quality service without delay or data loss could be provided through the Optical Packet and Circuit Integrated Network ${ }^{[4]}$.

\subsection{Communication line}

While it was possible to configure the network without using the optical fibers that were already laid (field fiber), it was necessary to use the field fibers since fiber laying fell outside of our objective (scenario) of the demo experiment. However, renting the commercial optical fiber over long distances increased the cost burden. Therefore, we borrowed the R\&D testbed network that is called JGN2plus ${ }^{[5]}$ and incorporated it into the experimental system to obtain the practical communication distance. This allowed us to test the factors of communication instability and limitations that might occur in reality. Considering the geography and the convenience of this test bed, the use of JGN2plus was suitable for this demo experiment. The details will be explained in subchapter 5.1 along with the site of experiment.

\subsection{Node equipment and devices}

\subsubsection{Silicon photonics switch (AIST)}

Optical devices that have a capability of the low power consumption and integration in the network are attractive, and AIST is working on the development of the cross bar switch $^{\text {Term } 2}$ using the silicon photonics technology ${ }^{[6]}$. This switch was incorporated into the network node for the configuration that enabled the transmission of video data for the first time. Although the general-use PLC optical switch was employed as the switch for some of the communication nodes, this silicon photonics switch was incorporated since low power consumption and highly integrated multiple ports could be demonstrated.

\subsubsection{Current-injection-type silicon-based high-speed optical switch (Fujitsu)}

At the Fujitsu Laboratories, Ltd., the development of the small silicon-based optical switch that allows mixed integration with electrical circuit is being done for the future dynamic optical path network. Fujitsu achieved a high-performance device with extremely high optical confinement efficiency as well as small cross-sectional surface area, employing the nano wire rib waveguide transverse direction $\mathrm{p}-\mathrm{i}-\mathrm{n}$ diode structure. It also achieved the switching speed of ns with the lowest switching power in the world ${ }^{[7]}$. This switch was also incorporated into the network node for the demo experiment.

\subsubsection{Power-saving next-generation ROADM (NEC)}

The reconfigurable optical add/drop multiplexer (ROADM) is equipment that enables efficient operation of the super high-speed high-capacity optical transmission network, by combining the wavelength multiplexing method and path control. Currently, there is insufficient degree of freedom in the optical path setting in the node devices of the network in which ROADM is deployed. The equipment system that allows switching the transponder ${ }^{\text {Term } 3}$ stored in the node at arbitrary wavelength and direction is important. NEC Corporation developed the transponder aggregator ${ }^{\text {Term } 4}$ to increase the freedom of optical path setting, and this will ultimately improve the use efficiency of the transponder ${ }^{[8]}$.

\subsubsection{High-speed wavelength tunable laser (Trimatiz)} Since it is necessary to be able to dynamically switch the wavelength in the future optical networks, the achievement of high speed in wavelength switching will be the key. Trimatiz, Ltd. is developing the wavelength tunable light source device based on the wavelength tunable laser diode (T-LD) that allows switching of the wavelength at millisecond or less. This technology allows the single T-LD to be tuned stably at high speed and a device that allows $5 \mathrm{GHz}$ resolution tuning of the C-band ${ }^{\text {Term } 5}$ was achieved. This was installed in the input end of the NEC's ROADM equipment, to create a configuration that allowed the switching of the wavelength.

\subsubsection{Optical amplifier}

The optical amplifier (Erbium-doped fiber amplifier: EDFA $^{\text {Term } 6}$ ) must be used to compensate the loss of the optical fiber communication line. The key point is to determine where in the network this device should be placed and the position is determined by the loss information on the optical fiber corresponding to the distance. In the optical path network, since the wavelength is switched dynamically, the EDFA must be capable of following the transient response ${ }^{[9]}$, and therefore, we selected the amplifier which has a suppressing technology of the transient response.

\subsubsection{Parametric arbitrary wavelength converter (Furukawa Electric and AIST)}

The wavelength converting technology is essential to effectively utilize the wavelength resource in the future optical path network. Since the coherent wavelength conversion using the highly nonlinear fiber (HNLF) can maintain the state of the light phase after conversion, in principle, it is not dependent on the data modulation format or modulation speed $^{[10]}$. Other than HNLF, there is the semiconductor 
optical amplifier method that can be used as the wavelength conversion technology, but HNLF was employed since the non-dependency on the modulation format would be useful in the future. In the demonstration, the wavelength conversion from the C-band to the L-band ${ }^{\text {Term } 5}$ was incorporated.

\subsubsection{High-speed autonomous-control optical tunable dispersion compensator (Furukawa Electric and AIST)}

Furukawa Electric Co., Ltd. and AIST proposed and demonstrated the tunable dispersion compensator that combines the wavelength dependent dispersion medium and the wavelength conversion by parametric process, called the optical parametric tunable dispersion compensator (P-TDC) $)^{[1]}$. By using the four wavelength mixing (FWM) of the $\mathrm{HNLF}^{[12]}$ that has low dispersion slope with the principle of phase maintaining wavelength conversion, we achieved a gridless broadband function surpassing $1 \mathrm{THz}$ that could not be achieved by the conventional tunable dispersion compensation technology. Projecting that the optical path will be dynamically switched in the future optical network, the high-speed tuning response of microsecond order is realized ${ }^{[13]}$. For the transmission experiment, this technology was applied to the field fiber of $105 \mathrm{~km}$ line through which the Super Hi-Vision signals are transmitted at $43 \mathrm{~Gb} / \mathrm{s}$.

\subsection{Terminal equipment}

\subsubsection{SHV transceivers (NHK)}

The Japan Broadcasting Association (Nippon Hoso Kyokai = NHK) leads the development of the device that enables the transmission and reception of uncompressed Super Hi-Vision (SHV) in the $24 \mathrm{~Gb} / \mathrm{s}$ dual green method by the $43 \mathrm{~Gb} /$ s optical signal $^{[14]}$. The network was configured via the $105 \mathrm{~km}$ field fiber. The long distance transmission was achieved by the high-speed autonomous control tunable dispersion compensation technology described in section 4.3.7.

\subsubsection{Content archive server, delivery server, and display}

The content archive server is the computer in which the image contents are stored, and this archive/delivery server functions by the command (section 4.1.1) of the network storage resource manager of the optical path network. The display was configured by devices that were readily available using the SHV monitor.

\subsection{Contents}

The contents we used were the SHV videos (NHK) and HiVision videos. The former was SHV videos with 33 million pixels/frame, and these were about four times larger both horizontally and vertically than the regular Hi-Vision videos. With the cooperation of NHK, two SHV videos were set in the demonstration system, ready to be transmitted.

For the regular Hi-Vision (or HD), we purchased a general-use HD video camera and shot our own contents. The videos were set in the delivery server.

\section{Demonstration experiment}

Importance of demo experiment for communication technologies is basically to demonstrate that it is possible to deliver information from point $\mathrm{A}$ to point $\mathrm{B}$, including the switching technology. The important factors of the experiment include the site where the transmitting/receiving terminals are located, construction of the experimental system based on the plan, and then to execute the transmission and switching of the actual video information. Considering these factors, we configured the demo experiment as shown in Fig. 3. The elemental technologies described in chapter 4 are also shown in the diagram. In this chapter, we describe how the elemental technologies described in the previous chapter were incorporated into the experiment.

Figure 3 shows the configuration where the two networks are mutually connected. The blue area on the left side is the NICT's Optical Packet and Circuit Integrated Network, and the green area on the right is the AIST's optical path network. NHK's SHV transceiver technology is set in the middle. This topology ${ }^{\text {Term } 7}$ was the result of discussing the points of mutual connection. The blue connection line indicates the optical fiber. The switch request method of the networks was predetermined. The network in the black dotted line on the right of the diagram were located entirely in Akihabara, and the line went to NICT's Optical Packet and Circuit Integrated Network via Otemachi, Koganei and then back to Otemachi, with connecting points NICT-EAST and NICT-WEST. After the control environment of the network was set up, and the stage progressed to the video distribution experiment (subchapter 5.2).

\subsection{Site of the experiment and construction of the experimental system}

Although it seemed that the demo experiment could be executed anywhere, we decided the site as we narrowed down the available filed optical fiber. Since it was necessary to use the R\&D testbed network (JGN2plus) as mentioned in subchapter 4.2, we considered the appropriate terminal station. The east terminal of the JGN2plus was located in Otemachi (Chiyoda-ku, Tokyo). Therefore, we reached the conclusion that we could create the network relatively easily by connecting with the AIST Akihabara (Soto-Kanda, Chiyoda-ku Tokyo) through the commercial optical fiber. Although it was also possible to connect to the AIST headquarters in Tsukuba, Ibaraki, the more convenient AIST Akihabara was selected since there was no extension of the R\&D testbed network to Tsukuba, and we wanted to conduct the demo experiment during the symposium. The distance between Otemachi and Akihabara was $1 \sim 2 \mathrm{~km}$ by line of sight, but the optical fiber actually used was $9.8 \mathrm{~km}$. This was because the usable laid optical fiber traveled up to a certain floor of the building and then went into the underground common utility hole. After the optical fiber line between Otemachi and Akihabara was 
set, it was connected to NICT since the west terminal of JGN2plus was located at NICT (Koganei). This range was set up as the network. The distance between these points was about $42.7 \mathrm{~km}$, and the round trip distance was $105 \mathrm{~km}$ from Akihabara. The demo site was located in two places, NICT (Koganei) and AIST (Akihabara). This distance was suitable as a network model connecting the area within a city. The major issue was to establish the communication by the $43 \mathrm{~Gb} / \mathrm{s}$ optical modulation signal, the fastest signal used in the demo. Therefore, we planned the complete compensation for the effect of signal degradation by wavelength dispersion in the $105 \mathrm{~km}$ of optical fiber, using the high-speed autonomous control tunable dispersion compensator that we developed. However, since the transmission loss at $0.2 \mathrm{~dB} / \mathrm{km}$ or more in the optical fiber and the loss due to optical connectors and parts were unknown, it was uncertain whether the optical $\mathrm{S} / \mathrm{N}$ ratio would fall within the acceptable range of the receiver. Therefore, we prepared a backup plan to decrease the difficulty of the transmission for the video distribution demo based on the simplification of the topology.

The site of the demo experiment was set up on one of the floors of AIST Akihabara, as shown in Fig. 4. The hardware was placed in groups by elemental technologies. Although it was compared with Fig. 3, in practice, the design in Fig. 4 was completed first. As a result of giving priority to the size of the device and convenience of connection in a limited space, the layout was determined without inconvenience. As the actual hardware was prepared as in Fig. 4, the devices and equipment were connected by each function block. In building the experimental system, the connection process was the most important, and misconnection or major communication line loss were not acceptable. The connections were done accurately and at appropriate signal level using a switch port table.

\subsection{Video distribution experiment}

One of the highlights of the demo experiment was to see whether the path communication for $43 \mathrm{~Gb} / \mathrm{s}$, the fastest optical modulation signal, was possible over the $105 \mathrm{~km}$ transmission distance. This is the communication path shown as a red line in Fig. 3. When the path was tested by bit error rate tester, it did not become error free even when the optical signal intensity was raised, and we were concerned about the disturbances or interruptions in the SHV video. However, when the actual connection was made with the receiver with the appropriate optical power, the communication was established due to the signal error correction function of the device. Although the signal error correction function was nothing special, whether the $43 \mathrm{~Gb} / \mathrm{s}$ path at transmission

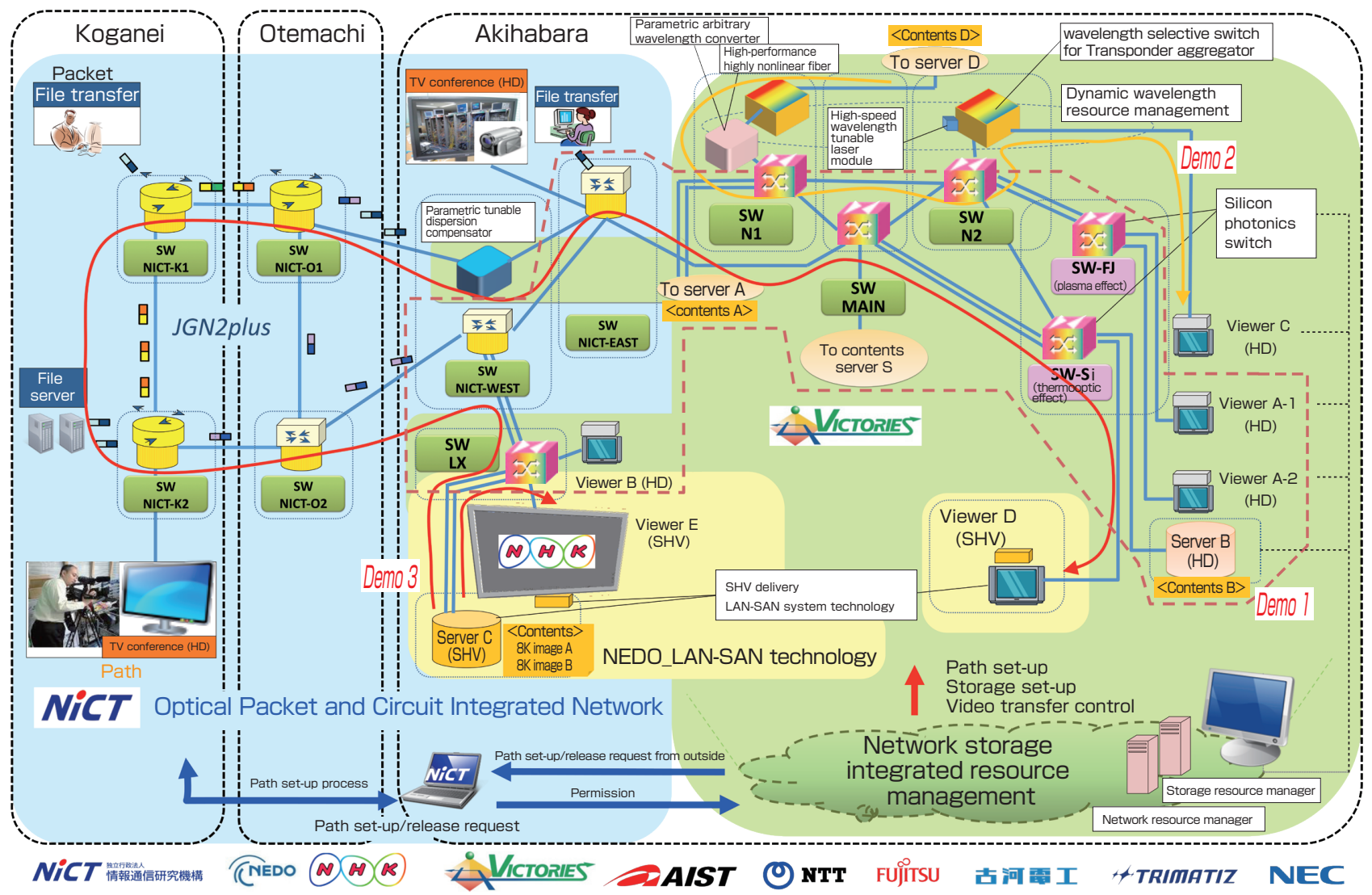

Fig. 3 Schematic diagram of the joint connection experiment for the optical network 
distance $105 \mathrm{~km}$ would fall into the error correctable range could only be confirmed by the experiment, and this was a major point of the video distribution experiment.

After the long-distance, high-speed communication path was established, the issue shifted to how to create the scenes that would be viewed in the public demonstration experiment. This was discussed carefully with NTT that handled the network storage/resource management technology. We called this the demo scenario. In conducting a large-scale demonstration experiment network, the significance of the elemental technologies would be diminished unless we create a demo scenario that showed all the elemental technologies actually in function. The executed demo scenario consisted of the reservation of the image and its control including the storage management equipment, the protection including the node device image, the cooperation with the NICT Optical Packet and Circuit Integrated Network, and the reserved distribution of SHV.

The demo experiment was conducted at the 3rd Vertically Integrated Center for Technologies of Optical Routing toward Ideal Energy Savings (VICTORIES) Symposium held on August 25, 2010. Table 2 shows the flow of the demo scenario. The network was configured so all the elemental technologies would cooperate and be presented in about 30 minutes. Looking at both the demo scenario and Fig. 3, in Demo 1, it was demonstrated that the content request from viewer A-1 and A-2 were answered based on the network storage integrated resource management, and the HD video was transmitted with the optimization of the server in which the contents were stored and the optical path that linked to the viewer. In Demo 2, in addition to the format for distributing

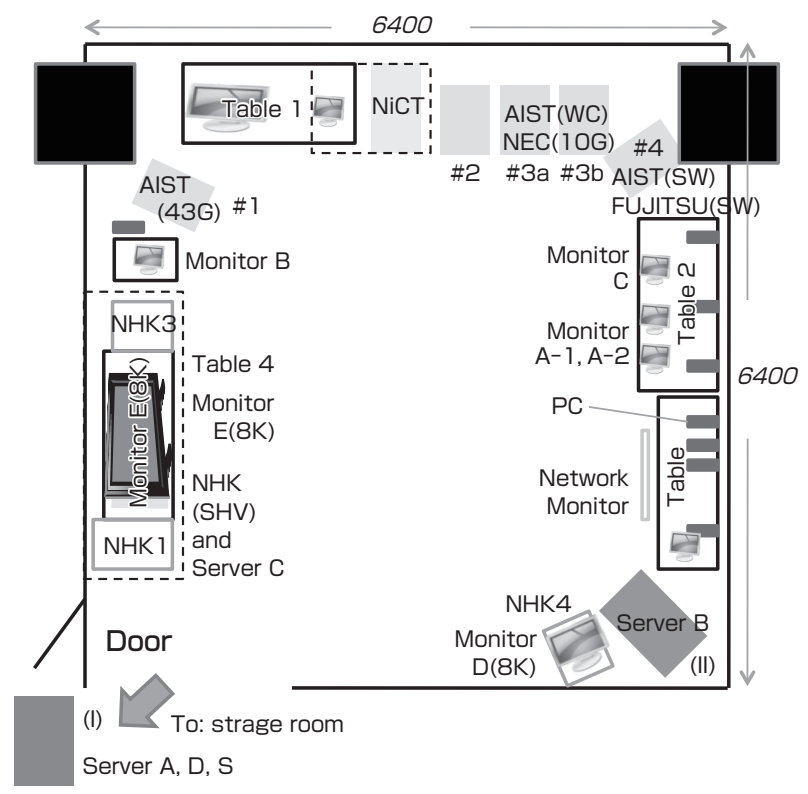

Fig. 4 Layout of the Akihabara site
Table 2. Demo scenario and elemental technologies

\begin{tabular}{|c|c|c|}
\hline & Demo scenario & Elemental technologies \\
\hline \multirow{4}{*}{$\begin{array}{l}\frac{1}{3} \\
\frac{0}{4}\end{array}$} & $\begin{array}{l}\text { Demo 1: Reservation of the video and its control including } \\
\text { the storage management device }\end{array}$ & $(a),(e),(j),(n),(p)$ \\
\hline & Demo 2: Protection (next-generation ROADM technology) & (e), (h), (i), (j) \\
\hline & NICT Demo: Optical Packet and Circuit Integrated Network & (b). (c). (d) \\
\hline & $\begin{array}{l}\text { Demo 3: SHV video distribution by reservation (NHK) } \\
\text { dissimilar netwrork connection, silicon optical switch, } \\
\text { tunable dispersion compensation }\end{array}$ & $\begin{array}{l}\text { (b), (c), (d), (e), (f), } \\
(\mathrm{g}),(\mathrm{j}),(\mathrm{k}),(\mathrm{l}),(\mathrm{m}),(\mathrm{o})\end{array}$ \\
\hline
\end{tabular}

contents D in server D as requested by viewer $\mathrm{C}$ via the dynamic wavelength resource manager, the protection action in case of transponder failure was demonstrated. In Demo 3, it was confirmed that the SHV video information could pass heterogeneous networks without any problem, by setting the optical path including the silicon photonics switch and long-distance transmission and by estimating the SHV video request from viewers B and D. Figure 5 shows the actual open demonstration. The three small monitors shown in the center corresponds to viewers $\mathrm{C}, \mathrm{A}-1$, and A-2 from left. The large screen on the right of the three small monitors is the network storage integrated resource management screen.

\section{Evaluation of the results and future developments}

It was confirmed in the experiment that all of the elemental technologies cooperated using the demo scenario of subchapter 5.2. The three points mentioned in chapter 3 were confirmed: A) to execute the multiple communication rates on the network, to manage them on the network, and to distribute the contents by switching the network according to the requested service; B) to check that the communication can be established by compensating the nonlinear responses from the field optical fiber, and to check that the different networks configured by different elements can be mutually connected; and C) to achieve low power consumption.

The power consumption measurement of the specific network nodes was conducted while in function, and Fig. 6 shows the measured and the projected values. Assuming that the switch ports and nodes were set up for the same number of devices used in the demo experiment using the core router, it was

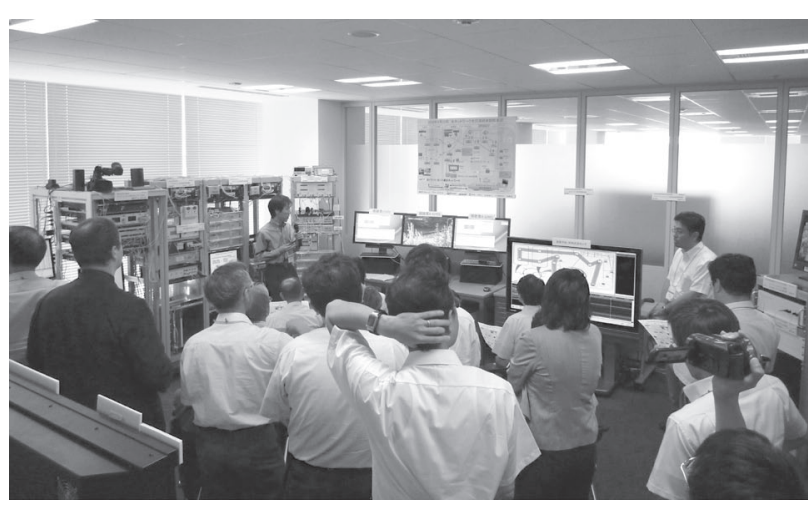

Fig. 5 Open demo experiment 
estimated to be about $13.4 \mathrm{~kW}$ including the optical amplifier. In the demo experiment, the power excluding the server was $1.5 \mathrm{~kW}$ with room for expandability to $\mathrm{Tb} / \mathrm{s}$. Since the power of some office appliances that could not be isolated from the measured power source circuit was included, the figure was slightly larger than the sum of the ratings of the device groups. However, it was found that basically, the energy saving effect on the value of the power consumption would be more significant as the high-capacity communication data were stuffed. Specifically, when the transmission rate per port becomes $100 \mathrm{~Gb} / \mathrm{s}$ as the silicon photonics optical switch is realized, it is estimated that power efficiency 1,000 times higher may be achieved. Considering the increased communication demand such as for HD video expected in the future, we believe sufficient decrease of power consumption was achieved, as we are able to slow down the increase of power consumption. The optical path network can contribute to the achievement of low power consumption of the communication network.

There were several things that became clear for the first time after starting to build the optical path network. Viewing from the OSI model ${ }^{\text {Term } 8}$, which is the basis of network connection, the first point is that the cooperation between the layers became complex. This is a complex involvement, starting from the switching occurring at the physical layer to the moment of display as the cache of the browser stores some of the images. We spent time establishing the communication in each optical path, such as in the line that used the media converter $^{\text {Term } 9}$, because the linkage function corresponding to the converter function behaved differently from the command of the resource management device. In the demo experiment, the issues related to the inter-layer cooperation were solved one by one to bring the system to function. We believe we need an opportunity for more specific demo experiments and mutual connections including the standardization of connection, in order to maximize the potential of the optical path network. Such findings could not have been obtained without the "vertical integration (collaboration)" where the technologies from devices to applications were convened and executed.

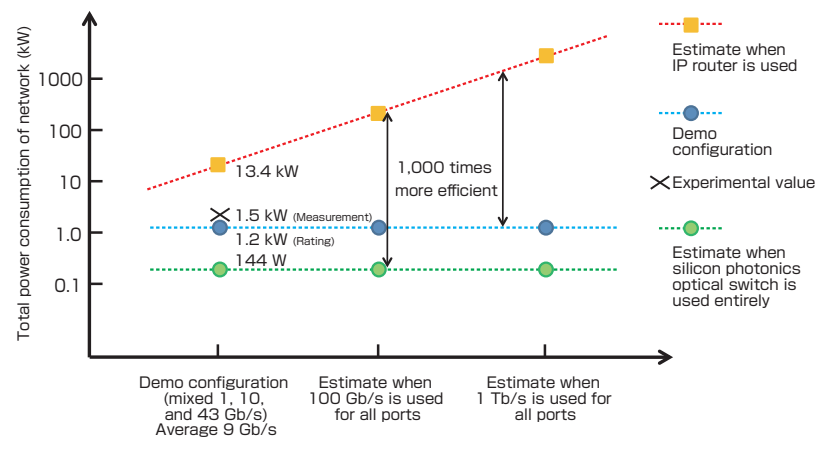

Fig. 6 Communication capacity and power consumption (including estimated values) in the demo configuration
In the future, it will be necessary to enhance the inter-layer cooperation and to compensate the differences in the wavelength and performances of the transceiver devices in the optical path network. Such R\&Ds will be continued as the optical path conditioning technology at the Network Photonics Research Center of AIST.

\section{Conclusion:the significance of the demo experiment}

A new optical communication network that supports the high-definition high-capacity video era was demonstrated through the joint efforts of AIST, five IT related companies, NICT, and NHK Science and Technology Research Laboratories. It was highly significant that through the vertical integration (collaboration) that crossed the organizations, the potential of the new network was demonstrated in surpassing the limitations of the power consumption and communication capacity of the devices that configured the current network. We were able to realize some of the aspects of the new video information service by incorporating the elemental technologies for the newly developed optical communication, and we believe this was an important step in the evolution of the optical communication technology.

\section{Acknowledgements}

In conducting the demo experiment we wish to express gratitude to the major contributions of NICT and NHK. We thank the people of the collaborating companies, whose efforts led the demo experiment to success. We also thank the researchers of AIST who were in charge of the elemental technologies, led by Tomohiro Kudoh, group leader of the Information Technology Research Institute, AIST, who worked to install and start up the demo. We would also like to mention that this demo experiment would not have been possible without the guidance and support of Shingo Ichimura, vice-president, AIST and Hiroshi Ishikawa, director, Network Photonics Research Center, AIST.

\section{Terminologies}

Term 1. Planar light wave circuit (PLC) optical switch: PLC often refers to the planar quartz waveguide.

Term 2. Cross bar switch: the switch with two switching state, the bar and cross states, when considering the connection port with two inputs and two outputs. This electric switching method was originally used in the telephone switchboard before digital switching became common. Recently, it refers to the internal switch element that dynamically selects the route when the data is exchanged between the CPU and the memory within a device. 
Term 3. Transponder: optical transmitting/receiving equipment that communicates at an arbitrary wavelength, by storing the client signal and converting it into frame format (such as OTN) appropriate for the backbone transmission networks.

Term 4. Transponder aggregator: a device that resolves the ROADM's issue of the limitation of wavelength and path in the optical route setting, and provides the degree of freedom in reconfiguring the optical route between the transmission line and the optical transmitting/receiving device (transponder). It is composed of the waveguide with wavelength selectivity and an optical matrix switch, and it enables the optical route setting where the arbitrary transponder connects to an arbitrary optical fiber transmission route at an arbitrary wavelength.

Term 5. C-band, L-band: the wavelength range used in optical communication. Conventional (C) band has the wavelength range of 1530-1565 nm, while long (L) band has the range $1565-1625 \mathrm{~nm}$.

Term 6. Erbium-doped optical fiber amplifier: a device that amplifies the optical signal directly in the fiber without the electrical signal conversion, utilizing the principle where the optical signal of a certain range is amplified when the excitation light of a certain wavelength is applied to a fiber doped with rare earth element erbium.

Term 7. Topology: a term that indicates the position and connection. In general, it indicates the connection format of the computer network. Star-type and ring-type network topology are most commonly used.

Term 8. Open systems interconnection (OSI): the standardization and organization where the communication protocol is broken down into the seven-layer structure. It is known as the OSI reference model.

Term 9. Media converter: a device that connects the different transmission media and converts the signals mutually. The most common one is the device that converts the LAN cable (copper wire) to optical fiber.

\section{References}

[1] Ministry of Internal Affairs and Communications, 2010 White Paper on Information and Communications in Japan, (White paper), (2010).

[2] S. Namiki, T. Kurosu, K. Tanizawa, J. Kurumida, T. Hasama, H. Ishikawa, T. Nakatogawa, M. Nakamura and K. Oyamada: Super HD video transmission and extremely green optical networks for future, IEEE Journal of Selected Topics in Quantum Electronics, 17 (2), 446-457 (2011).

[3] K. Yamada, Y. Tsukishima, K. Matsuda, M. Jinno, Y. Tanimura, T. Kudoh, A. Takefusa, R. Takano and T. Shimizu: Joint storage-network resource management for super high-definition video delivery service, Optical Fiber Communication (OFC) 2011, Conference on, JWA1, (2011).

[4] H. Harai: Report of proof-of-concept experimental and live demonstration of optical packet \& circuit integrated network over JGN2plus optical-fiber testbed, IEICE technical report, 110, 7-12 (2010).

[5] NICT, Advanced Testbed Network for R\&D, http://www.jgn. nict.go.jp/.

[6] Y. Shoji, K. Kintaka, S. Suda, H. Kawashima, T. Hasama and H. Ishikawa: Low-crosstalk 2 x 2 thermo-optic switch with silicon wire waveguides, Optics Express, 18, 9071-9075 (2010).

[7] S. Sekiguchi, T. Kurahashi, K. Kawaguchi and K. Morito: Current-type silicon-based optical switch with silicon germanium waveguide, IEEE Photonics Society 23rd Annual Meeting, WW3 (2010).

[8] M. Sakauchi, I. Nishioka, S. Nakamura, T. Chu and Y. Urino: Demonstration of fast optical protection in ROADM system with one-chip color/direction- independent add/drop multiplexer employing silicon photonic circuit, Optical Fiber Communication (OFC) 2009, Conference on, JThA51 (2009).

[9] Y. Oikawa, Y. Horiuchi, Y. Tanaka, M. Shiga, N. Shiga and H. Nagaeda: Super-fast AGC-EDFA for the burst-mode systems without gain excursion in 20-ns and 21-dB ramped input, 2008 Conference on Optical Fiber Communication/ National Fiber Optic Engineers Conference, 1-3 (2008).

[10] S. Petit, T. Kurosu, S. Namiki, M. Takahashi and T. Yagi: Truly arbitrary wavelength conversion by cascaded four-wave mixing in low dispersion slope sbs suppressed highly nonlinear fibers, 2010 IEEE Photonics Society Winter Topicals Meeting Series, 115-116 (2010).

[11] S.Namiki: Wide-band and -range tunable dispersion compensation through parametric wavelength conversion and dispersive optical fibers, Journal of Lightwave Technology, 26, 28-35 (2008).

[12] M. Takahashi, R. Sugizaki, J.Hiroishi,M.Tadakuma,Y. Taniguchi and T.Yagi: Low-loss and low-dispersion-slope highly nonlinear fibers, Journal of Lightwave Technology, 23, 36153624 (2005).

[13] K. Tanizawa, J. Kurumida, H. Ishida, Y. Oikawa, N. Shiga, M. Takahashi, T. Yagi and S. Namiki: Microsecond switching of parametric tunable dispersion compensator, Optics Letters, 35, 3039-3041 (2010).

[14] T. Nakatogawa,S. Okabe,M. Nakamura,K. Oyamada,F. Suginoshita, T. Ikeda and K. Shogen: Wireless and fibre-optic live contribution link for uncompressed super hi-vision signals, The Best of IET and IBC, IET Journals, 2, 31-36 (2010).

\section{Authors}

\section{Junya KURUMIDA}

Researcher, Optical Signal Processing System Research Team, Network Photonics Research Center (NPRC), AIST. He received the master's degree from the Graduate School of Engineering, Osaka Electro-Communication University in 1998 and the doctorate from the Interdisciplinary Graduate School of Science and Engineering, Tokyo Institute of Technology.

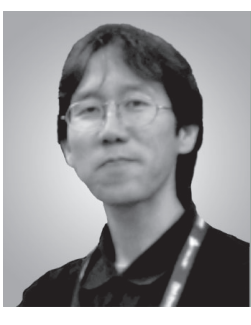
Doctor (Engineering). He worked at the Fujitsu Ltd., Photonics Research Institute of AIST, and University of California, Davis. He is with NPRC since 2009, and engages in R\&D for device and system in the optical fiber communication technology field. Cooperated with Shu Namiki to oversee the entire demo experiment and managed the operation and wrote all chapters of this paper. 


\begin{abstract}
Shu NAMIKI
Leader, Optical Signal Processing System Research Team, Network Photonics Research Center, AIST. He received the master's degree from the Graduate School of Science and Engineering, Waseda University in 1988. Joined the Furukawa Electric Co., Ltd. in 1988. Engaged in research and product development of the semiconductor optical device, mode-locking laser, light amplifier, nonlinear fiber optics, and optical transmission. Visiting researcher at Massachusetts Institute of Technology, USA from 1994-1997. Joined AIST in 2005. Has coauthored over 200 publications, and served as member of several international conference programs, as well as member of International Organization for Standardization/International Electrotechnical Commission (ISO/IEC). Vice-chairman of the Editorial Board, IEICE Transaction B (Communications) of the Institute of Electronics, Information and Communication Engineers. Associate editor, Optics Express, Optical Society of America from 2005. Currently, advisory editor, Optics Express and deputy chief editor of IEICE Society. Doctor of Science. Fellow of the Optical Society of America. In this paper, considered the direction of the demo experiment and the outline of the elemental technology to be combined.
\end{abstract}

\section{Discussions with Reviewers}

\section{Background of the paper}

Comment (Naoto Kobayashi, Center for Research Strategy, Waseda University)

The main theme of this paper is that a demonstration experiment was conducted to realize the dynamic optical path network. Yet I think there is lack of explanation on what is a dynamic optical path network, and how it can realize low-power optical communication (also why that's not possible with packet communication). I think you should describe these to help the understanding of readers outside of the field.

Answer (Junya Kurumida)

I think you indicated an important point in the description. To clarify the optical path network, I added the following section in paragraph of chapter 2: “... a new network called the 'optical path network' that directly links the users with optical path, and engages in R\&D to solve the above issues. Particularly, the network where the optical network is switched flexibly to actively maintain the optical path in response to the high-capacity information request is called the "dynamic optical path network'." Also, as the reason for the realization of lowpower optical communication, I added in chapter 2: "These efforts will enable the high-speed data communication without going through multiple electric signal processing, and a network that handles large-capacity information at low power can be configured."

To make the point clear for the readers, I added the following sentence in chapter 1 and the issues will be discussed in the following chapter: "On the other hand, there is a major problem in the sustainable development of such advanced communication service. It will be explained in the following chapter."

Comment (Hiroshi Tateishi, New Energy and Industrial Technology Development Organization)

I understand that the point of this paper is the demonstration of the low power consumption by using only optical components in the high-capacity network, the seamless mutual connection technology of heterogeneous networks and the implementation of the super high-capacity content distribution based on these technologies, in one model experiment. However, I cannot understand where the breakthrough point was, or how things were solved. Please organize and present the background and the outline of the research in an understandable manner. The press release* for this experiment on the AIST website is much clearer. After doing this, please describe the important points from the perspective of R\&D. You have described what you have done in detail, but you did not explain "why they had to be done" or "what were the problems and how they were solved". Please consider these and write with a stance that you are trying to get some third-party people to understand.

Answer (Junya Kurumida)

Thank you very much for your important indication. I added the segment "...the new network technology that overcomes the limitations of power consumption and communication capacity of the devices that configured the current network" in the outline, and added the sentence "With the background of increased demand in communication mainly for the Internet, the energy savings in optical communication is an immediate issue". in the beginning of "1 Introduction" to clarify the background and outline of the research.

To solidify the perspective from R\&D, I added the description about establishing the $43 \mathrm{~Gb} / \mathrm{s}$ path communication, that is the highlight of this demo and is the fastest optical modulation signal at $105 \mathrm{~km}$ transmission distance, to the "5.2 Video distribution experiment". The three viewpoints of the scenario explained in chapter 3 were organized as A, B and C and were explained correspondingly in chapter 6 .

*http://www.aist.go.jp/aist_j/press_release/pr2010/pr20100824_2/ pr20100824_2.html

\section{Demo experiment system and its significance Comment (Naoto Kobayashi)}

I think the elemental technologies are explained well. However, the part on the achievement of the demo experiment system and the significance of the successful research are expressed weakly, and the significance of this major accomplishment may not be communicated to the readers. Therefore I think you should do the following:

1. You should clearly show how the scenario shown in chapter 3 was realized in the demo experiment, as the result shown in chapter 3, "(1) to configure a network with coexisting communication elemental technologies such as $1 \mathrm{~Gb} / \mathrm{s}$ and $10 \mathrm{~Gb} / \mathrm{s}$, (2) to configure the network using the already laid optical fiber, and (3) to work on these further to achieve low power consumption".

2. Since it is highly significant that the elemental technologies were integrated to create a system, I think you should tell the difficulties of the integration and how you were able to overcome them in more detail.

\section{Comment (Hiroshi Tateishi)}

In subchapter 5.1, you describe in detail the distance of the intermediate optical fiber, but you do not explain what that length means for the experiment, so I don't know how to interpret the information. Do you wish to say that in practice, implementing this distance is sufficient for the purpose of demonstration, or did it simply end up this way and there's not much technological meaning? Whichever the reason, when you give specific figures, the readers become curious about the meaning behind them. Answer (Junya Kurumida)

Thank you very much for indicating the improvement points for the paper. 
1. The three viewpoints explained in chapter 3 , were clarified as $\mathrm{A}, \mathrm{B}$ and $\mathrm{C}$, with the introduction "we shall discuss the issue from the three perspectives" I matched them to the explanation in chapter 6 .

2. Since I provided a specific figure for the distance of the optical fiber line in subchapter 5.1, I added the story about the difficulty of high-speed communication over distance: "This distance was suitable as a network model connecting the area within a city. The major issue was to establish the communication by the $43 \mathrm{~Gb} / \mathrm{s}$ optical modulation signal, the fastest signal used in the demo. Therefore, we planned the complete compensation for the effect of signal degradation by wavelength dispersion in the $105 \mathrm{~km}$ of optical fiber, using the high-speed autonomous control tunable dispersion compensator that we developed. However, since the transmission loss at $0.2 \mathrm{~dB} / \mathrm{km}$ or more in the optical fiber and the loss due to optical connectors and parts were unknown, it was uncertain whether the optical $\mathrm{S} / \mathrm{N}$ ratio would fall within the acceptable range of the receiver. Therefore, we prepared a backup plan to decrease the difficulty of the transmission for the video distribution demo based on the simplification of the topology."

In response to the above, I described the important point that we overcame in the demo experiment in subchapter 5.2: "One of the highlights of the demo experiment was to see whether the path communication for $43 \mathrm{~Gb} / \mathrm{s}$, the fastest optical modulation signal, was possible over the $105 \mathrm{~km}$ transmission distance. This is the communication path shown as a red line in Fig. 3. When the path was tested by a bit error rate tester, it did not become error free even when the optical signal intensity was raised and we were concerned about the disturbances or interruptions in the SHV video. However, when the actual connection was made with the receiver with appropriate optical power, the communication was established due to the signal error correction function of the device. Although the signal error correction function was nothing special, whether the $43 \mathrm{~Gb} / \mathrm{s}$ path at transmission distance $105 \mathrm{~km}$ would fall into the error correctable range could only be confirmed by the experiment, and this was a major point of the video distribution experiment."

\section{Vertical integration (collaboration) and its concept Comment (Naoto Kobayashi)}

I think the "vertical integration (collaboration)" is one of the most valuable points in this research, and I think you should include a simple explanation along with a figure.

Answer (Junya Kurumida)

Thank you for your indication. I added Fig. 1 and the explanation for the figure as follows: "Figure 1 shows the conceptual diagram. To utilize the optical switch in the network, a mechanism for controlling the optical switch according to the network application is necessary. That is to say, a mechanism is needed where the mutual connections are made between the HD video server and display, teleconference systems are handled, and these are appropriately managed according to the optical path and storage information. Moreover, vertical integration (collaboration) is essential to introduce the silicon optical path switch and wavelength resource management technologies, and to consolidate and realize the technologies that are developed individually, from device level to application level."

\section{Evaluation of the result Comment (Hiroshi Tateishi)}

Chapter 6 is an important part for Synthesiology, but the description is insufficient. What are the meanings of the problems that occurred in the experiment and how were they solved? Moreover, what significance do they have in the future development? As of now, you're saying, "When we did the experiment, many unforeseen problems occurred, but we could not solve all of them on the spot". It is merely a report of the results without much information for the reader.

Also, there is no evaluation for "power consumption 1.5 kW". When I see Fig. 6, I can understand that it can be reduced further compared to the electric signal processing, but you need the explanations for, "to which extent you have to decrease in practice?" and "were the results sufficient or insufficient?"

\section{Answer (Junya Kurumida)}

I added the description on the specific problems that occurred in the experiment and the solution process in the beginning of the video distribution experiment in subchapter 5.2. I added the process of establishing the communication with $43 \mathrm{~Gb} / \mathrm{s}$ path, the optical modulating signal with highest speed in this demo, with $105 \mathrm{~km}$ transmission distance.

Also, in the beginning of chapter 2, I added the detailed description of the communication traffic and electricity: "Assuming that this trend will continue for about 20 years, the traffic will be about 1,000 times higher. This calls for the improvement of power efficiency." Building on this comment, I added the following section as the evaluation of the power consumption in chapter 6: "Assuming that the switch ports and nodes were set up for the same number of devices used in the demo experiment using the core router, it was estimated to be about $13.4 \mathrm{~kW}$ including the optical amplifier. In the demo experiment, the power excluding the server was $1.5 \mathrm{~kW}$ with room for expandability to Tb/s." and "Specifically, when the transmission rate per port becomes $100 \mathrm{~Gb} / \mathrm{s}$ as the silicon photonics optical switch is realized, it is estimated that power efficiency 1,000 times higher may be achieved. Considering the increased communication demand such as for HD video expected in the future, we believe sufficient decrease of power consumption was achieved, as we are able to slow down the increase of power consumption." 